\title{
Belphégor
}

\section{Le survival comme mode d'appréhension de}

l'Histoire

Enjeux narratifs et génériques du survival historique

\section{Sébastien Fevry}

\section{(2) OpenEdition}

\section{Journals}

Édition électronique

URL : https://journals.openedition.org/belphegor/3763

DOI : 10.4000/belphegor.3763

ISSN : 1499-7185

Éditeur

LPCM

Référence électronique

Sébastien Fevry, «Le survival comme mode d'appréhension de l'Histoire », Belphégor [En ligne], 19-1 | 2021, mis en ligne le 25 juin 2021, consulté le 09 juillet 2021. URL : http://journals.openedition.org/ belphegor/3763 ; DOI : https://doi.org/10.4000/belphegor.3763

Ce document a été généré automatiquement le 9 juillet 2021.

\section{cc) (†) $\odot$}

Belphégor est mis à disposition selon les termes de la Licence Creative Commons Attribution - Pas d'Utilisation Commerciale - Pas de Modification 4.0 International. 


\section{Le survival comme mode d'appréhension de l'Histoire}

Enjeux narratifs et génériques du survival historique

\section{Sébastien Fevry}

1 En cherchant à immerger leurs spectateurs.rices au cœur de l'événement, certains films historiques contemporains adoptent un mode d'appréhension de l'Histoire qui s'apparente au survival entendu en un sens large comme une situation narrative où des personnages luttent pour leur survie. Je proposerai ici d'étudier selon cette perspective trois films correspondant à cette intersection entre film historique et film de survie, à savoir La Peur (2015) du Français Damien Odoul, Dunkirk (2017) de l'Anglais Christopher Nolan et Le Fils de Saul (2015) du Hongrois László Nemes ${ }^{1}$. Bien que ces films traitent de sujets historiques différents (la Guerre 14-18, l'Opération Dynamo menée par les Anglais en 1940, les camps d'extermination durant la Seconde Guerre mondiale), ils ont pour point commun d'envisager l'événement passé comme une expérience de survie en milieu hostile et de proposer une vision embarquée du conflit où le/la spectateur/rice partage presque uniquement la vision d'un ou plusieurs personnages saisis au centre de l'action.

2 Partant de ce constat, cet article souhaiterait problématiser davantage l'articulation entre film historique et survival tant d'un point de vue narratif que du point de vue de la réception critique des productions, puisqu'on peut notamment se demander si le choix de plonger au plus près de l'événement ne conduit pas paradoxalement à couper celui-ci de son contexte social et historique. En recourant aux apports croisés de la narratologie et des approches génériques, l'article aura comme propos d'éclaircir le statut du survival avant d'envisager la façon dont ce dernier intervient dans le champ $\mathrm{du}$ film historique et les conséquences qu'il implique dans l'appréhension des faits du passé, aussi bien en termes d'expériences esthétiques que de recadrages discursifs. 


\section{Le survival : du (sous-)genre à la modalité}

3 Curieusement, si l'on tient compte de la popularité du terme, il existe peu d'ouvrages ou d'articles consacrés au survival en tant que genre ou sous-genre filmique. Dans un ouvrage collectif sur les genres du cinéma hollywoodien, Thomas Sobchack l'associe au film d'aventure, lequel est compris comme une vaste catégorie générique où « mankind is depicted as living in a world fraught with perils of all kinds, but through the exemplary effort, cooperation, and bravery of individuals and groups, the social order is (...) maintained against all threats to its stability $»^{2}$. Le survival apparait alors au côté d'autres (sous-)genres filmiques comme « the swashbuckler, the war film, safari/jungle epic $\rrbracket^{3}$, sans qu'il soit facile de déterminer si le survival interfère avec ces sous-genres ou s'il constitue un sous-genre à part entière. D'après sa définition la plus courante, le survival renvoie à un programme assez minimal, à savoir « un genre cinématographique dans lequel un ou plusieurs personnages font des efforts physiques pour survivre $\aleph^{4}$, ce qui explique pourquoi celui-ci s'entrecroise aussi aisément avec des genres filmiques possédant des caractéristiques thématiques plus élaborées, comme le film de cape et d'épée, la science-fiction ou le film historique ${ }^{5}$. Sous cet éclairage, La Peur, Le Fils de Saul et Dunkirk peuvent aisément se percevoir comme des survivals historiques, puisqu'ils décrivent chacun la lutte pour la survie d'un individu ou d'un groupe d'individus dans un environnement historique hostile, qu'il s'agisse de la guerre des tranchées, du camp d'Auschwitz ou d'une plage bombardée par des avions allemands.

D'un point de vue générique, cette définition du survival me parait toutefois limitée par une approche taxinomique qui hésite entre autonomiser le survival comme un genre à part entière ou le reléguer comme sous-genre de catégories génériques mieux définies. Dans les deux cas, l'approche consiste à envisager le survival de façon hiérarchique en délimitant son territoire et ses affiliations, dans un système générique conçu de façon pyramidale. Cette approche échoue à prendre en compte ce qui fait précisément l'intérêt du survival, à savoir sa grande plasticité, son extraordinaire pouvoir transgénérique et transmédiatique. Comme on l'a déjà signalé, le survival peut s'allier à d'autres genres filmiques comme le film d'aventure, le film de guerre, la science-fiction, le film d'horreur ou certaines écofictions décrites par Christian Chelebourg ${ }^{6}$. Par ailleurs, le survival ne désigne pas simplement un (sous-)genre filmique, mais il permet aussi de caractériser certains types de jeux vidéo et de programmes de divertissement (Koh-Lanta, You vs the Wild sur Netflix, par exemple), sans compter les affinités que ce genre entretient avec des courants de pensée comme le survivalisme ${ }^{7}$.

5 Par conséquent, faire du survival une sous-catégorie ou même une catégorie à part entière reviendrait à ignorer cette plasticité et à isoler le survival au sein de sousensembles définis, alors qu'il faut peut-être davantage penser le survival non comme une sous-catégorie, mais comme une modalité traversant les frontières de genres mieux établis en proposant un même type d'expérience esthétique, quel que soit l'univers thématique dans lequel cette expérience s'incarne.

6 Une approche moins cloisonnante des genres a été théorisée par Christine Gledhill dans un l'article "Rethinking genre" paru en 2000. Pour Gledhill, qui travaille principalement sur le mélodrame, il faut certes imaginer qu'il existe un système des genres correspondant globalement à un ensemble de règles et d'attentes partagées par les producteurs/rices et les spectateurs/rices, mais que ce système soutient et produit des modalités génériques qui peuvent passer d'un genre à l'autre en fournissant 
certains types d'expériences esthétiques. À propos du mélodrame, elle écrit que son efficacité historique peut être conçue de deux façons interdépendantes : «first, as an early cultural machine for the mass production of popular genres capable of summoning up and putting into place different kinds of audience; second, as a modality, understood as a culturally conditioned mode of perception and aesthetic articulation $»^{8}$. Pour Gledhill, le concept de modalité permet de comprendre comment le système générique est pourvu d'un mécanisme « of 'double articulation', capable of generating specific and distinctively generic formulae in particular historical conjunctures, while also providing a medium of interchange and overlap between genres $»^{9}$.

7 Dans cette perspective, la modalité générique, « like register in socio-linguistic, defines a specific mode of aesthetic articulation adaptable across a range of genres, across decades, and across national cultures $»^{10}$. La modalité ne doit pas tant se concevoir en termes de contenus, qu'il s'agisse de thématiques, de décors ou de personnages-types, que comme une capacité d'agencement permettant, pour le mélodrame du moins, d'organiser «the disparate sensory phenomena, experiences, and contradictions of a newly emerging secular and atomising society in visceral, affective and morally explanatory terms $»^{11}$.

8 Par son caractère décloisonnant, l'approche de Gledhill me semble particulièrement indiquée pour traiter le cas du survival, même si, dans ce cas précis, il est clair que nous n'avons pas affaire à une 'machine générique' aussi puissante que le mélodrame et qu'il faudrait sans doute définir plus précisément les conditions socio-historiques qui amenèrent à maturation les caractéristiques du survival ${ }^{12}$. Cela étant, dans le cadre de cet article, ma proposition sera de considérer le survival comme une modalité au sens de Gledhill, c'est-à-dire comme une forme d'articulation esthétique pouvant passer d'un genre à l'autre, rendant compte de certaines données expérientielles et amenant une certaine appréhension de l'Histoire quand elle s'actualise dans le genre du film historique.

\section{Le survival comme simulation d'un récit en mimèsis I}

La question se pose dès lors de savoir comment définir le type d'expérience esthétique procurée par le survival, en dépassant la simple description qui consisterait à le catégoriser sur la base de son seul noyau thématique, c'est-à-dire la survie d'un ou plusieurs individus dans un environnement hostile. En partant de certains films contemporains présentés comme des survivals exemplaires ${ }^{13}$, tels que The Revenant (2015) d'Alejandro González Iñárritu et Gravity (2013) d'Alfonso Cuarón, il semble que l'on puisse définir la modalité du survival par un ensemble de traits stylistiques récurrents visant à transmettre au public un sentiment de confusion et de désorientation, ces traits apparaissant également dans les films historiques envisagés, qu'il s'agisse de La Peur, du Fils de Saul ou de Dunkirk.

D'un point de vue cinématographique, les films survivals sont souvent guidés par une logique d'immersion, avec une caméra embarquée qui adopte le point de vue du personnage principal. Le lieu de l'action (un espace naturel, mais pas nécessairement) constitue un environnement au sens littéral du terme dans la mesure où il encercle le personnage ou le groupe de personnages, ce qui se concrétise à l'écran par des mouvements de caméra panoramiques à 360 degrés permettant au public de prendre la 
mesure de cet espace circulaire au centre duquel se trouvent les protagonistes. On peut aussi observer que le survival met fréquemment en scène des personnages mutiques dont l'expérience avec le monde environnant s'effectue sur un mode corporel, dans un registre propre au cinéma haptique décrit par Laura Marks ${ }^{14}$.

Dans le cadre de cet article, je voudrais faire valoir que cet ensemble de traits stylistiques s'associe étroitement avec une certaine modalité de narration qui est celle d'un récit (ou, plus précisément, de la simulation d'un récit) qui correspondrait à la mimèsis I. Déjà chez Ricœur, la mimèsis I, en tant que préfiguration narrative, renvoie à l'expérience temporelle brute, avant toute saisie par l'action configurative, une expérience que Ricœur décrit comme "confuse, informe et, à la limite, muette $»^{15}$, ce qui fait déjà singulièrement écho à l'expérience esthétique du survival, d'autant que Ricœur insiste également sur le fait que cette mimèsis I se caractérise par un rapport d'intra-temporalité qui rend compte de «la condition d'être jeté parmi les choses ${ }^{16}$, le sujet étant alors saisi dans l'épaisseur du temps.

12 Mais c'est surtout la relecture que propose Raphaël Baroni de la triple mimèsis qui permet d'asseoir l'idée que la narrativité du survival tend à correspondre à des récits simulant la mimèsis I, c'est-à-dire à des récits qui se réfèrent à des événements inachevés, ancrés dans le présent et non rétrospectifs où évoluent des sujets engagés dans l'histoire au moment où elle se raconte. Cette relecture de la triple mimèsis ricœurienne - qui prend comme point de départ «l'opposition entre une narrativité en prise directe avec l'événement et une narrativité rétrospective et configurée par autrui $»^{17}$ - permet de doter la modalité narrative du survival d'un statut plus précis que celui d'un simple récit immersif. Chez Baroni, le récit immersif correspond en effet à une intrigue "conçue dans le but d'immerger le lecteur dans une expérience simulée et de nouer une tension orientée vers un dénouement éventuel $\aleph^{18}$, une telle définition pouvant aussi bien valoir pour des récits qui se déroulent au rythme des événements (simulation de mimèsis I) que pour des récits rétrospectifs à visée intrigante (mimèsis II intrigante).

13 En plaçant le survival sous le signe d'une simulation d'un récit en mimèsis I, il s'agit donc de souligner que la modalité du genre s'exprime pleinement lorsque le cadre du récit se trouve ramené à un cercle réduit (unité de temps, de lieu et d'action) et que, plus fondamentalement, pour reprendre les termes de Baroni, « les plans diégétique et déictique sont indiscernables $~^{19}$, puisque «le 'je' du narrateur et le 'je' du protagoniste sont reliés dans l'épaisseur temporelle d'un sujet incarné $»^{20}$. Toutefois, là où Baroni envisage encore la présence d'un sujet « en train de vivre l'histoire qu'il (se) raconte »" le survival, dans ses déclinaisons audiovisuelles en tout cas, peut effacer toute trace d'énonciation et donner l'impression au/à la spectateur/rice d'épouser le point de vue d'un personnage vivant l'expérience de la survie, sans que se décèle, dans le film ou la série, la présence d'une action racontante.

En ce sens, la modalité générique du survival pousse à l'extrême la simulation d'un récit en mimèsis I, à tel point qu'on peut se demander si les déclinaisons les plus radicales du survival présentent encore la visée intrigante que Baroni identifie comme l'une des grandes formes de mise en intrigue. En effet, le suspense à l'œuvre dans le survival reste souvent élémentaire (le personnage va-t-il survivre ou non ?). Par ailleurs, cet effet de suspense peut s'émousser à mesure que le personnage prolonge son immersion dans un univers donné, ce qui explique que de nombreux films comme Gravity maintiennent l'intérêt $d u / d e$ la spectateur/rice en ponctuant la trame principale par des événements 
inattendus (le surgissement d'une météorite par exemple) agissant comme autant de surprises, sans pour autant que ces micro-événements participent à tendre ou à ramifier les nœuds de l'action principale. D'autres films sont marqués par une extension de la durée (Gerry [2002] de Gus Van Sant) ou par des séquences relativement longues (Into the Wild [2007] de Sean Penn) dans lesquelles la tension narrative faiblit au profit d'une focalisation presque sémiotique sur la tentative effectuée par le personnage pour déchiffrer les signes extérieurs d'un environnement non familier.

Aussi peut-on se demander, en dernière instance, si la modalité du survival n'aurait pas davantage à voir avec l'interprétation sémiotique du monde qu'avec l'action narrative en tant que telle, ce qui renvoie à ce que Ricœur disait de la mimèsis I en définissant celle-ci comme le lieu de la "pré-compréhension du monde de l'action: de ses structures intelligibles, de ses ressources symboliques et de son caractère temporel ${ }^{22}$. Plus finement alors, l'intérêt premier du survival ne serait pas tant de dramatiser l'action que d'entraver, suspendre ou différer la pré-compréhension de l'environnement extérieur, de rabattre la mise en intrigue au niveau de l'aventure sémiotique elle-même.

16 Sans entrer davantage dans ces considérations théoriques, ma proposition sera de considérer la modalité générique du survival comme étant la simulation d'un récit en mimèsis I retraçant la survie d'individus en milieu hostile. Tout l'enjeu du travail de la mise en scène (cinématographique, télévisuelle ou sérielle...) est alors de donner l'illusion que nous sommes de plain-pied avec le personnage et de reconduire chez le/la spectateur/rice les conditions perceptives qui seraient celles d'un sujet traversant une expérience de survie. Cette façon de considérer le survival participe à éclairer les enjeux de certains films historiques contemporains. Par le prisme de cette modalité, il devient possible de mieux comprendre l'historicité de ceux-ci, leur capacité à se connecter à d'autres genres filmiques et enfin les (re)cadrages discursifs qu'ils peuvent susciter.

\section{Une logique de l'avant}

17 Le premier enjeu qu'éclaire la modalité du survival a trait au statut temporel des films, à la place qu'ils occupent dans l'historiographie de tel ou tel événement du passé. Dans une première compréhension, on pourrait décrire le survival comme un genre de l'après, puisqu'il faut survivre après la catastrophe, après avoir été largué dans un environnement hostile ou éjecté dans l'espace. À première vue, l'enjeu des films se situe donc dans l'après-coup d'une suite d'actions ou de décisions condamnant les personnages à devoir évoluer dans un environnement hostile. Les films historiques en mode survival n'échappent pas à cette règle de l'après-coup : dans Dunkirk, lorsque le film commence, les personnages sont déjà encerclés sur la plage par les troupes allemandes; dans Le Fils de Saul, le personnage principal est déjà embrigadé dans les Sonderkommandos sans que le film ait montré un temps antérieur à cette période d'horreur; dans La Peur, le protagoniste se trouve plongé dans un conflit dont il ignore les causes et les conséquences, le scénario ne livrant que « des bribes d'information ${ }^{23}$.

Cependant, le fait de considérer ces représentations comme des simulations de récits en mimèsis I laisse entrevoir une dynamique temporelle qui relève presque d'un mouvement contraire, du moins si l'on se situe au niveau de l'acte d'énonciation des films. En effet, des réalisateurs comme Christopher Nolan, Damien Odoul ou László Nemes appartiennent à la troisième ou quatrième génération postérieure aux 
événements racontés, ce qui fait que leur travail s'apparente davantage à un travail de post-mémoire que de mémoire ${ }^{24}$. Dunkirk, La Peur et Le Fils de Saul arrivent à la fois après le travail des historiens et après les témoignages des contemporains de l'événement. Dans cette perspective, le fait de concevoir le survival historique comme la simulation d'un récit en mimésis I pourrait être interprété comme une volonté de faire un récit non pas de l'après, mais de l'avant. Il s'agirait de retrouver l'événement au plus près de son noyau expérientiel, avant qu'il ne soit reconfiguré par des discours surplombants qui viendraient le doter d'une explication a posteriori. Tout se passe comme si, à travers la modalité expérientielle du survival historique, l'objectif était de traverser les couches de discours qui s'étaient accumulées au-dessus de l'événement passé pour essayer de toucher celui-ci dans sa singularité vécue.

Dans une interview, Chrisopher Nolan confie qu'il lui semblait important de montrer le point de vue partiel et limité des soldats anglais coincés dans la poche de Dunkerque : "si vous vous retrouvez au cœur d'un événement, surtout à cette époque qui ne connaissait pas les smartphones, il est très difficile d'avoir une vue d'ensemble de la situation (...) C'est seulement dans les journaux qu'ils ont découvert la réalité de ce qu'ils avaient traversé $»^{25}$, dans les journaux, c'est-à-dire dans des récits de mimèsis II qui recadraient déjà l'événement en le dotant d'un sens plus large. Dans Sortir du noir, le bref essai consacré au Fils de Saul, Georges Didi-Huberman note qu'avec ce film, nous n'aurons pas « à notre disposition 'un tableau de la situation' à Auschwitz-Birkenau en 1944. Le film se contente - mais c'est considérable - d'accompagner le travail, la peur et la folle décision d'un seul personnage $»^{26}$. Dans La Peur, le cas est plus complexe puisque la bande sonore est régulièrement ponctuée par la lecture d'extraits de lettres écrites depuis le front, ce qui veut dire qu'un premier travail de prise de distance, entre mimèsis I et mimèsis II, s'élève depuis le monde chaotique véhiculé par les images.

Dans tous les cas, on s'aperçoit que l'enjeu pour ces films n'est nullement de répondre à la visée configurante traditionnellement attribuée au récit historique, lequel cherche "à 'comprendre' rétrospectivement l'histoire déjà dépassée " ${ }^{27}$. Au contraire, sur le mode du survival, les films historiques tendent à produire la simulation ou, mieux encore, le reenactement ${ }^{28}$ d'un événement historique donné, en intégrant dans le déroulement de l'action les tâtonnements et la confusion de ceux qui participèrent directement à l'événement.

\section{Un socle expérientiel commun}

21 Le deuxième intérêt de penser le survival non comme un (sous-)genre mais comme une modalité est de comprendre les effets de connexions et les rapprochements intertextuels (ou intericoniques ${ }^{29}$ ) qui peuvent s'opérer entre les films. En effet, à partir du moment où le survival est conçu comme la mise en place d'un programme expérientiel minimal (survivre), il n'y a rien d'étonnant à ce que des affinités se mettent en place entre des films relevant de genres différents, mais qui se focalisent de manière similaire sur la façon dont les personnages gèrent leur rapport à un environnement hostile. Par exemple, certaines scènes tirées de La Peur où l'on voit le héros traverser hagard de grands espaces désolés peuvent être rapprochées de certaines séquences du Revenant, film historique certes, mais dont l'action se déroule plus d'un siècle auparavant. Pareillement, la mise en scène d'un abri temporaire menacé par les éléments extérieurs se retrouve dans de nombreux genres (science- 
fiction, horreur...), l'important étant finalement moins la nature de l'abri (bateau, satellite, bus...) que le rapport à l'environnement qui se trouve exprimé de manière similaire en termes de gros plans et de hors-champs.

Si l'on considère le survival comme la modalité environnementale par excellence dans le sens où il focalise l'attention sur l'environnement immédiat du personnage, il n'est pas surprenant que certains survivals historiques soient amenés à susciter des interprétations attirant l'attention sur le rapport des protagonistes à leur environnement naturel. Dans sa critique du film La Peur, Thomas Sotinel souligne qu'un personnage du film " s'adapte merveilleusement à son nouvel écosystème " ${ }^{30}$, tout en faisant remarquer à propos du paysage qu'on ne sait plus « dans quel sens les arbres poussent, ni où commence le ciel " ${ }^{31}$. Dans certains cas, la direction interprétative envers l'environnement naturel peut être pointée par le réalisateur lui-même. Ainsi, aux yeux de Christopher Nolan, Dunkirk ne relève pas du genre historique, mais plutôt du survival: "c'est une évacuation. Une histoire de survie $»^{32}$. Le choix du terme 'évacuation' n'est pas anodin puisqu'il peut aussi bien recouvrir le déroulement d'une opération guerrière que le déplacement de populations à la suite d'un désastre écologique imminent. Nolan semble conscient de ce rapprochement, puisque son intérêt porte, dit-il, sur le comportement collectif qui " advient généralement lors des grandes catastrophes naturelles ou humaines " $^{33}$. L'utilisation du terme "survie " n'a donc pas pour but d'isoler Dunkirk dans le genre du film historique, mais plutôt, semble-t-il, de favoriser un mouvement de connexion entre une situation historique donnée et un contexte écologique contemporain dominé par l'existence d'un horizon herméneutique lié à l'ère de l'anthropocène et aux dégradations environnementales ${ }^{34}$.

Remarquons aussi que certains réalisateurs refusent explicitement de considérer leur film sous l'angle du survival, comme c'est le cas de László Nemes pour Le Fils de Saul : « Je ne voulais pas faire un film sur la survie, mais sur la réalité, sur la mort. Car la survie est un mensonge, c'était l'exception " ${ }^{35}$. Ce refus peut se comprendre à un premier niveau comme le refus d'un terme impropre à qualifier une situation historique où la survie était impossible ou extrêmement rare. À un second niveau, renvoyant davantage au contexte historiographique entourant les représentations filmiques de la Shoah, le refus de l'étiquette 'film de survie' peut s'entendre comme une volonté de préserver la singularité de l'expérience historique et d'empêcher la connexion avec d'autres genres filmiques comme le film-catastrophe ou le film d'aventure auxquels est habituellement associé le survival.

Plus largement, au-delà de ces cas spécifiques, on pourrait aussi soutenir que le survival, parce qu'il reproduit un même type d'expérientalité36 favorise, pour reprendre les termes de Gledhill, le développement d'un «culturally conditioned mode of perception ${ }^{37}$ pouvant opérer aussi bien en contexte historique que contemporain ou futuriste. Plus que telle ou telle scène, telle ou telle allusion, c'est une même modalité expérientielle que le/la spectateur/rice est amené à retrouver de film en film, cette modalité pouvant également entrer en résonance avec des expériences qui ne relèvent pas nécessairement du domaine cinématographique ou littéraire. Etant donné que le survival opère à un niveau premier de mimèsis qui est celui de l'expérience humaine élémentaire, dans le registre de l'intra-temporalité décrit par Ricœur, on peut estimer que le noyau expérientiel véhiculé par les représentations est capable de résonner avec des situations pré-narratives expérimentées par le spectateur/la spectatrice ou le/la cinéaste . Autrement dit, la simulation d'une pré-narrativité en mimèsis I aurait pour 
propriété de faciliter la mise en écho d'expériences qui n'auraient pas été encore reconfigurées par des récits (à vocation intrigante ou configurante) en mimèsis II.

Un tel phénomène s'illustre particulièrement avec une anecdote que raconte Nolan à propos de la genèse de Dunkirk. Bien que le film fasse preuve d'une réelle rigueur historique, il repose aussi sur une expérience personnelle du réalisateur. Nolan inscrit le véritable point de départ de son film dans une traversée de la Manche effectuée des années auparavant avec sa femme et un ami: on avait fait cette traversée "pour s'imprégner de cette épopée. C'est l'une des choses les plus difficiles, les plus effrayantes que j'aie vécues. J'ai été extrêmement soulagé d'arriver en un seul morceau alors même que personne ne nous bombardait. Il n'y avait que nous trois sur ce petit bateau, la mer et les éléments $»^{38}$. Par ce biais, on voit comment Nolan fonde son appréhension d'un événement historique sur une expérience physique personnelle. Le propos pour Nolan n'est pas d'assimiler ces expériences ou de prétendre qu'elles sont équivalentes. Il s'agit plutôt de les relier sur la base du socle expérientiel commun que constitue la survie dans un milieu (plus ou moins) hostile.

\section{Recadrages narratifs}

Le fait de penser certains films historiques sous le registre du survival permet enfin d'être plus précis quant aux recadrages que subissent ces films lorsqu'ils sont interprétés à l'intérieur du genre 'film historique', la notion de genre étant ici considérée classiquement comme une catégorie d'interprétation déterminant des attentes et reposant sur un certain nombre de conventions. Pour l'exprimer en termes genettiens ${ }^{39}$, si nous avons réfléchi précédemment en termes d'intertextualité, il s'agit maintenant de déplacer l'attention vers la catégorie de l'architextualité pour déterminer le type de réception affectant le film historique en mode survival, lorsque celui-ci est reçu au sein d'un périmètre générique donné.

Pour commencer, on peut estimer que le survival sacrifie la lisibilité d'un phénomène historique pour la remplacer par une suite d'expériences vives, coupées de tout contexte et de toute volonté de clarification. Dans cette perspective, le principal risque est que les survivals historiques suscitent des orientations de lecture annulant toute préoccupation historique, la situation de dégradation de l'environnement valant en quelque sorte pour elle-même. Ironiquement, Joshua Levine, pourtant conseiller historique sur Dunkirk, ne semble pas prendre conscience de ce risque lorsqu'il écrit que " filmer une menace sans visage libère l'événement de ses ramifications géopolitiques $»^{40}$. C'est à partir de ce risque de décontextualisation qu'il convient de positionner les différentes stratégies de réception opérant autour des films qui nous intéressent ici.

Qu'il s'agisse de Dunkirk, de La Peur ou du Fils de Saul, il s'avère que l'un des traits récurrents du travail critique consiste à questionner la dimension configurante qui manque à ces films et qui leur aurait permis de donner une portée rétrospective aux événements représentés à l'écran. Parfois, il s'agit simplement de déplorer un manque de contextualisation historique. Dans la critique du Monde à propos du film La Peur, Thomas Sotinel regrette, tout en saluant le parti pris esthétique du réalisateur, que « la logique infernale du conflit, les mécanismes de machine guerrière sont simplifiés à l'extrême $»^{41}$. Pour Le Fils de Saul - qui mériterait à lui seul une étude de réception -, si une grande partie de la critique salue la force d'innovation $\mathrm{du}$ film ${ }^{42}$, certaines voix 
discordantes se font entendre, comme la critique de Jean-Philippe Tessé dans Les Cahiers du cinéma, qui attaque le mode immersif mis en place par le film, un tel mode substituant « au témoignage la reproduction spectaculaire de ce sur quoi il porte, sur le mode 'vis ma vie' $»^{43}$. Parallèlement, certains critiques se posent la question de savoir dans quelle mesure un tel film pourrait être projeté à un public ignorant tout de l'histoire de la Shoah. Chris Beney, sur le site de Télérama, remarque que le film «ne peut pas marcher sur un spectateur amnésique ou ignorant de la Shoah mais y en a-t-il encore ? Le génocide fait-il suffisamment partie de notre histoire commune pour que le cinéma en montre autre chose ? $»^{44}$

Dans d'autres cas, la critique ne se contente pas de regretter une absence de contextualisation, mais complète narrativement ce que le film ne prend pas en charge. Ainsi, si l'on se penche sur les critiques de Dunkirk, on s'aperçoit que celles-ci commencent souvent par rappeler le contexte historique du film qui était à peine évoqué à l'écran. Par exemple, Studio entame sa critique par un long rappel des faits qui occupe une bonne part de l'article : « 21 mai 1940. Les armées alliées sont balayées du continent européen par la puissance de feu allemande... $»^{45}$ Même stratégie dans les Inrockuptibles : "Au cours de la 'drôle de guerre' et suite à la percée allemande dans le nord de l'Hexagone, les troupes françaises et anglaises ont dû battre en retraite vers la côte de la mer du Nord... ${ }^{46}$ De tels extraits sont intéressants à relever, car ils montrent comment la critique du film devient productrice d'une nouvelle mise en récit qui se déploie en reprenant la visée configurante du récit historique qui était imparfaitement accomplie par le film lui-même. De cette façon, l'activité critique participe à renforcer l'opération de genration ${ }^{47}$, non pas tellement en fournissant une interprétation sur le contenu du film, mais en entourant la narration première de récits cadres permettant son affiliation au genre du film historique.

En guise de conclusion, je souhaiterais revenir sur l'un des points théoriques principaux de cet article qui a consisté à croiser perspectives narratologique et générique en vue d'éclaircir le fonctionnement de certains films historiques contemporains. Sous un angle épistémologique, les approches de Gledhill (ou de Laporte) et les nouvelles narratologies auxquelles on peut affilier le travail de Raphaël Baroni peuvent être rapprochées quant à leur visée processuelle et dynamique. Ces approches saisissent leur objet - le genre ou le récit - non comme des catégories fixes qu'il s'agirait d'essentialiser ou dont il faudrait isoler le mode de fonctionnement, mais comme des processus de signification à l'œuvre dans des productions et des contextes spécifiques. Autrement dit, ces approches visent à saisir des dynamiques plutôt que des états en adoptant une perspective où il s'agit moins de comprendre ce qu'est le genre ou le récit que d'envisager les modalités d'action par lesquelles ces pratiques discursives informent les champs médiatique et culturel.

Sans nul doute, cette conjonction spécifique entre approches générique et narratologique mériterait d'être développée davantage, mais il apparaît déjà, à travers le cas du survival, que cette double approche se révèle particulièrement féconde pour saisir les enjeux de certains films historiques contemporains. Sur le versant générique, l'attention s'est portée sur les effets de connexion que favorisait la modalité générique $\mathrm{du}$ survival, laquelle permet de décloisonner et d'interroger les frontières entre genres en produisant des effets de rapprochement et d'interconnexion qui peuvent être encouragés ou refusés par les instances de production. Sur le versant narratif, le fait de concevoir le survival comme la simulation d'un récit en mimèsis I a permis d'étendre 
l'enquête narrative au-delà des bornes d'un récit donné pour suivre les récits seconds qui anticipent, prolongent ou recadrent la narration principale, faisant ainsi des films les centres de constellations narratives au sein desquelles gravitent aussi bien les récits véhiculés par la critique que les anecdotes personnelles rapportées par les réalisateurs.

Dans tous les cas, qu'il s'agisse de Dunkirk, La Peur ou Le Fils de Saul, le travail a montré comment, sur fond d'événements historiques pourtant différents, s'élève une même modalité expérientielle qui, par son extrême récurrence dans le champ médiatique contemporain, constitue un mode culturel de perception à part entière, repérable dans d'autres films, associée à d'autres genres, et qui pourrait faire sens plus largement dans le contexte écologique actuel. Cela étant, demeure à la fin de cet article l'interrogation portant sur les conditions historiques, culturelles et sociales qui transforment peu à peu un programme expérientiel narratif minimal, cantonné dans certains genres emblématiques comme le film-catastrophe, en une modalité générique capable d'articuler une vaste gamme de récits médiatiques renvoyant aussi bien à des événements passés, présents ou à venir.

\section{NOTES}

1. On pourrait aussi ajouter à cette liste le récent 1917 (2019) de Sam Mendès.

2. Ma traduction : «l'humanité est représentée comme vivant dans un monde rempli de périls de toutes sortes, mais grâce aux efforts, à la coopération et au courage exemplaires d'individus et de groupes, l'ordre social est (...) maintenu contre toutes les menaces à sa stabilité ». Thomas Sobchack, "The Adventure Film », dans Wes D. Gehring (dir.), Handbook of American Film Genres, New York/Westport, Connecticut/London, Greenwood Press, 1988, p. 9-10.

3. Ma traduction: «le film de cape et d'épée, le film de guerre, l'épopée dans la jungle ou le safari ». Ibid., p. 9.

4. Je reprends volontairement la définition du survival donnée par Wikipédia dans l'article consacré au film de survie. À ce sujet, voir https://fr.wikipedia.org/wiki/Film_de_survie (consulté le 8 janvier 2020).

5. Dans la suite de cet article, la dénomination « film historique » sera préférée à celle de « film de guerre ». Le caractère plus englobant $d u$ film historique permet de recouvrir les trois films examinés, à la différence de la catégorie «film de guerre » qui échoue à définir génériquement $L e$ Fils de Saul. Pour une définition de ces deux genres, voir Vincent Pinel, Écoles, genres et mouvements au cinéma, Paris, Larousse, 2000.

6. Christian Chelebourg, Les Écofictions. Mythologies de la fin du monde, Bruxelles, Les Impressions nouvelles, 2012.

7. Dans le cadre de cet article, je maintiendrai une nette distinction entre le survival comme expérience esthétique et le survivalisme en tant que courant de pensée apparu aux Etats-Unis dans les années 70, consécutivement au premier choc pétrolier. Pour une approche socioculturelle du survivalisme, voir Bertrand Vidal, Survivalisme. Êtes-vous prêts pour la fin du monde ?, Paris, Arkhe éditions, 2018.

8. Ma traduction : «premièrement, comme une machine culturelle pour la production en masse de genres populaires capable dans les premiers temps d'attirer et de retenir différents types de public; deuxièmement, comme une modalité, comprise comme un mode de perception et 
d'articulation esthétique conditionné culturellement ». Christine Gledhill, « Rethinking Genre », dans Christine Gledhill et Linda Williams (dir.), Reinventing Film Studies, London, Arnold, 2000, p. 227.

9. Ma traduction: «de "double articulation", capable de générer des formules génériques spécifiques et distinctes dans des conjonctures historiques particulières, tout en fournissant également un environnement d'échange et de chevauchement entre les genres ». Ibid., p. 229.

10. Ma traduction : " comme le registre en sociolinguistique, définit un mode spécifique d'articulation esthétique adaptable à travers une gamme de genres, à travers les décennies, et à travers les cultures nationales ». Ibid.

11. Ma traduction : «Les phénomènes sensoriels disparates, les expériences et les contradictions d'une société nouvellement émergente et atomisée en termes viscéraux, affectifs et moralement explicatifs ». Ibid., p. 228-229.

12. On pourrait émettre l'hypothèse que le survival, dans son acception contemporaine, se constitue en tant que machine générique dans les années 70, en profitant à la fois de la vague des films-catastrophes hollywoodiens comme La Tour infernale (Guillemin, Allen, 1974) et de l'émergence du survivalisme en tant que courant de pensée lié aux conséquences du premier choc pétrolier.

13. De nombreux sites Internet fournissent la liste des 40,165 ou 254 meilleurs films de survie... À titre d'exemple, voir la liste fournie par Sens Critique: https://www.senscritique.com/liste/ Survivals_les_films_de_survie/485673 (consulté le 8 janvier 2020).

14. Laura U. Marks, The Skin of the Film. Intercultural Cinema, Embodiment, and the Senses, Durham and London, Duke University Press, 2000.

15. Paul Ricœur, Temps et récit. L'Intrigue et le récit historique, 1, Paris, Seuil, coll. « Points Essais ", 1983, p. 12.

16. Ibid., p. 121.

17. Raphaël Baroni, L'CEuvre du temps. Poétique de la discordance narrative, Paris, Seuil, 2009, p. 85.

18. Raphaël Baroni, «Face à l'horreur du Bataclan : récit informatif, récit immersif et récit immergé ", Questions de communication [En ligne], 34, 2018, p. 114. http:// journals.openedition.org/questionsdecommunication/15659 (consulté le 8 janvier 2020).

19. Raphaël Baroni, L'Euvre du temps, p. 87.

20. Ibid.

21. Ibid.

22. Paul Ricœur, Temps et récit. L'Intrigue et le récit historique, 1, op. cit., p. 108.

23. Thomas Sotinel, «La Peur : la mort en bleu horizon », Le Monde, 12 septembre 2018, https:// www.lemonde.fr/televisions-radio/article/2018/09/12/la-peur-la-mort-en-bleuhorizon_5353703_1655027.html (consulté le 8 janvier 2020).

24. Pour Marianne Hirsch, la post-mémoire «describes the relationship that the "generation after" bears to the personal, collective, and cultural trauma of those who came before - to experiences they "remember" only by means of the stories, images, and behaviors which they grew up ". Marianne Hirsch, The Generation of Postmemory. Writing and Visual Culture After the Holocaust, New York, Columbia University Press, 2012, p. 5.

25. Joshua Levine (propos recueillis par), «Entretien avec Christopher Nolan», dans Joshua Levine, Dunkerque. Dans les coulisses du film de Christopher Nolan, Paris, HarperCollins France, 2017, p. 19.

26. Georges Didi-Huberman, Sortir du noir, Paris, Éditions de Minuit, 2015, p. 31.

27. Raphaël Baroni, L'Euvre du temps, op. cit., p. 89.

28. Au sujet de cette notion, voir notamment Anne Bénichou, «Introduction. Le reenactment ou le répertoire en régime intermédial ", Intermédialités. Refaire / Redoing, 28-29, automne 2016printemps 2017, p. 26-27. Pour une analyse de Dunkirk selon cette perspective, voir Rémy Besson, 
« Dunkerque, une reconstitution historique ? ", Mémoires en jeu, 6, 2018, https://www.memoiresen-jeu.com/actu/dunkerque-une-reconstitution-historique/ (consulté le 23 juin 2021).

29. Mathilde Arrivé, «L'intelligence des images - l'intericonicité, enjeux et méthodes », E-rea [En ligne], 13.1 | 2015, mis en ligne le 15 décembre 2015, consulté le 22 décembre 2020. URL : http:// journals.openedition.org/erea/4620 ; DOI : https://doi.org/10.4000/erea.4620.

30. Thomas Sotinel, « La Peur : la mort en bleu horizon », art. cit..

31. Ibid.

32. Joshua Levine (propos recueillis par), « Entretien avec Christopher Nolan », art. cit., p. 16.

33. Igor Hansen-Love (propos recueillis par), «Dunkerque. Nolan monte au front », dans Studio Ciné Live, 91, août 2017, p. 37.

34. Pour Kate Rigby, notre principal «horizon herméneutique " est dorénavant celui du changement climatique et de l'épuisement des ressources naturelles. Au sujet de cette expression, voir Kate Rigby, citée dans Andreas Malm, L'Anthropocène contre l'histoire. Le réchauffement climatique à l'ère du capital, Paris, La Fabrique, 2017, p. 151.

35. Fabien Lemercier (propos recueillis par), « Pas un film sur la survie, mais sur la réalité, sur la mort », https://cineuropa.org/fr/interview/292995/ (consulté le 8 janvier 2020).

36. Pour Monika Fludernik, l'expérientialité se définit comme «the quasi-mimetic evocation of real-life experience ». Monika Fludernik citée par Caracciolo, dans «Experientiality ", The living handbook of narratology, https://www.lhn.uni-hamburg.de/node/102.html (consulté le 8 janvier 2020).

37. Christine Gledhill, « Rethinking Genre », art. cit., p. 227.

38. Joshua Levine (propos recueillis par), «Entretien avec Christopher Nolan », art. cit., p. 15.

39. Gérard Genette, Palimpsestes. La littérature au second degré, Paris, Seuil, 1982.

40. Joshua Levine, Dunkerque. Dans les coulisses du film de Christopher Nolan, op. cit., p. 341.

41. Thomas Sotinel, « La Peur : la mort en bleu horizon », art. cit..

42. Le film reçoit le Grand Prix au Festival de Cannes en 2015 et l'Oscar du meilleur film en langue étrangère à la cérémonie des Oscars en 2016.

43. Jean-Philippe Tessé cité sur Allociné, http://www.allocine.fr/film/fichefilm-237178/ critiques/presse/ (consulté le 8 janvier 2020).

44. Chris Beney, "Le Fils de Saul est une fiction, mais il m'a fallu 6 mois pour le comprendre ", Télérama'vodkaster, 13 novembre 2015, http://www.vodkaster.com/actu-cine/fils-de-seulliberation-polemique-nemes-royer-lanzmann-huberman/1275859 (consulté le 8 janvier 2020).

45. Igor Hansen-Love, « Dunkerque. Nolan réinvente le film de guerre avec un chef-d'œuvre radical », Studio Ciné Live, 91, août 2017, p. 79.

46. Alexandre Büyükodabas, « Nolan remporte la bataille de Dunkerque », Les Inrockuptibles, 1129, juillet 2017, p. 14.

47. Selon Chloé Delaporte, l'opération de genration d'un film renvoie au «processus sémiotique qui construit les objets en tant que films en leur assignant des qualités conçues comme intrinsèques". Chloé Delaporte, Le Genre filmique. Cinéma, télévision, Internet, Paris, Presses Sorbonne Nouvelle, 2015, p. 120. 


\section{RÉSUMÉS}

En cherchant à immerger les spectateurs.rices au cœur de l'événement, certains films historiques contemporains adoptent un mode d'appréhension de l'Histoire qui s'apparente au survival entendu en un sens large comme une situation narrative où des personnages luttent pour leur survie. Sur la base de l'analyse des films La Peur (Odoul, 2015), Le Fils de Saul (Nemes, 2015) et Dunkirk (Nolan, 2017), cet article problématise l'articulation entre film historique et survival tant d'un point de vue narratif que du point de vue de la réception des productions. L'article envisage la façon dont le survival, en tant que modalité générique, intervient dans le champ du film historique et les conséquences que cela implique dans l'appréhension des faits du passé, aussi bien en termes d'expériences esthétiques que de recadrages discursifs.

INDEX

Mots-clés : survival, histoire, cinéma, narration, genre, mimèsis

\section{AUTEUR}

\section{SÉBASTIEN FEVRY}

Sébastien Fevry est professeur à l'Ecole de Communication de I'Université catholique de Louvain (UCLouvain) et coordinateur du GIRCAM. II travaille dans le champ des Memory Studies et des cultures visuelles. En 2021, il coordonne avec Emmanuelle Fantin et Katharina Niemeyer l'ouvrage collectif Nostalgies contemporaines. Médias, culture et technologie. II est par ailleurs l'auteur de nombreux articles dans des revues comme Image \& Narrative, Studies in French Cinema, Memory Studies, Intermédialités... 